\title{
TOO MUCH LOVE, a Root Regulator Associated with the Long-Distance Control of Nodulation in Lotus japonicus
}

\author{
Shimpei Magori, ${ }^{1}$ Erika Oka-Kira, ${ }^{1}$ Satoshi Shibata, ${ }^{2}$ Yosuke Umehara, ${ }^{2}$ Hiroshi Kouchi, ${ }^{2}$ \\ Yoshihiro Hase, ${ }^{3}$ Atsushi Tanaka, ${ }^{3}$ Shusei Sato, ${ }^{4}$ Satoshi Tabata, ${ }^{4}$ and Masayoshi Kawaguchi ${ }^{1}$ \\ ${ }^{1}$ Department of Biological Sciences, Graduate School of Science, The University of Tokyo, 7-3-1 Hongo, Bunkyo-ku, \\ Tokyo 113-0033; ${ }^{2}$ Plant Physiology Department, National Institute of Agrobiological Sciences, 2-1-2 Kannondai, Tsukuba, \\ Ibaraki 305-8602, Japan; ${ }^{3}$ Radiation-Applied Biology Division, Quantum Beam Science Directorate, Japan Atomic Energy \\ Agency, 1233 Watanuki-machi, Takasaki, Gunma 370-1292, Japan; ${ }^{4}$ Kazusa DNA Research Institute, 2-6-7 Kazusa-kamatari, \\ Kisarazu, Chiba 292-0818, Japan
}

Submitted 12 October 2008. Accepted 25 November 2008.

Legume plants tightly control the development and number of symbiotic root nodules. In Lotus japonicus, this regulation requires HAR1 (a CLAVATA1-like receptor kinase) in the shoots, suggesting that a long-distance communication between the shoots and the roots may exist. To better understand its molecular basis, we isolated and characterized a novel hypernodulating mutant of $L$. japonicus named too much love $(\mathrm{tml})$. Compared with the wild type, $\mathrm{tml}$ mutants produced much more nodules which densely covered a wider range of the roots. Reciprocal grafting showed that tml hypernodulation is determined by the root genotype. Moreover, grafting a harl shoot onto a $\mathrm{tml}$ rootstock did not exhibit any obvious additive effects on the nodule number, which was further supported by double mutational analysis. These observations indicate that a shoot factor $H A R 1$ and a root factor TML participate in the same genetic pathway which governs the long-distance signaling of nodule number control. We also showed that the inhibitory effect of TML on nodulation is likely to be local. Therefore, TML may function downstream of $H A R 1$ and the gene product TML might serve as a receptor or mediator of unknown mobile signal molecules that are transported from the shoots to the roots.

Rhizobium-legume symbiosis is one of the most successful mutually beneficial interactions on earth. In this symbiosis, soil bacteria collectively called rhizobia supply the host legumes with ammonia produced through bacterial nitrogen fixation, in contrast to host plants providing the rhizobia with their photosynthetic products. To accomplish this biotic interaction, leguminous plants develop root nodules in which to confine rhizobia and exchange nutrients with the symbiotic partner. This novel lateral organ differentiation (i.e., nodulation) is triggered by Nod factors (NF), lipochito-oligosaccharide signal molecules secreted by rhizobia. Upon NF perception, legumes activate a signaling cascade composed of multiple regulatory factors. This signaling cascade initiates a series of morphological changes in the roots (Geurts et al. 2005; Riely et al. 2004; Stacey et al. 2006). One of the earliest observable changes takes place at the root hairs. The tips of the root hairs start to swell, branch, and finally curl to trap rhizobia in the curling pockets. Subsequently, from this initial infection site, the

Corresponding author: Masayoshi Kawaguchi;

E-mail: masayosi@biol.s.u-tokyo.ac.jp plants induce tube-like structures called infection threads, which pack rhizobia inside and grow toward the nodule primordium in a host-controlled manner. In parallel, the activation of the NF signaling cascade also results in dedifferentiation of the cortical cells and induction of cortical cell division, leading to nodule primordium formation. Once the infection threads reach to the nodule primordia, the rhizobia are endocytosed into the plant cells and become nitrogen-fixing bacteroids. Recent studies utilizing two model legumes, Lotus japonicus and Medicago truncatula, have been making great strides in better understanding of these sequential processes at the molecular level. In fact, many of the positive regulators, including the putative NF receptor kinase genes NFRI and NFR5, have been identified (Madsen et al. 2003; Radutoiu et al. 2003), augmenting our knowledge of the molecular mechanisms that promote nodule development.

On the other hand, negative regulatory mechanisms to repress nodulation have long been postulated but their molecular basis remains elusive. Because nodule development is an energetically expensive process for host plants, overproduction of nodules should be circumvented. Therefore, such negative regulation of nodulation appears to be of great importance. It is well known that the plant hormone ethylene functions as a negative regulator in early infection events such as infection thread formation or elongation and cortical cell activation (Sugawara et al. 2006). In many legume species, including L. japonicus, it has been shown that exogenous application of ethylene or its immediate precursor has resulted in a decreased number of nodules, whereas inhibitors of ethylene biosynthesis enhance nodule formation (Nukui et al. 2000). Consistent with the proposed inhibitory role of ethylene in nodulation, an ethylene-insensitive mutant sickle of M. truncatula and transgenic L. japonicus plants expressing the mutated ethylene receptor gene both produce an increased number of infection threads as well as nodules or nodule primordia (Nukui et al. 2004; Penmetsa and Cook 1997). In addition, endogenous ethylene is also important for providing a positional cue for nodule organogenesis. In Pisum sativum, the mRNA of the 1-aminocyclopropane-1-carboxylate (ACC) oxidase, which catalyzes the ethylene precursor ACC to form ethylene, is expressed exclusively in the cell layers opposite the phloem poles (Heidstra et al. 1997). As a result, nodule primordium development is repressed in the vicinity of the phloem poles by the effect of ethylene; instead, nodule primordia are predominantly formed opposite the xylem poles (Heidstra et al. 1997). The same nodule position control is likely to be conserved in M. truncatula because nodules of the ethylene-insensi- 
tive sickle mutants are randomly distributed, whereas those of wild-type plants are developed mostly opposite the xylem poles (Penmetsa et al. 2003).

In addition to ethylene-mediated control of nodulation, legume plants are proposed to utilize a negative feedback regulation in which early infection events systemically and rapidly repress further nodule formation on younger root regions (Kosslak and Bohlool 1984; Malik and Bauer 1988; Nutman 1952; Pierce and Bauer 1983). This autoregulatory signaling enables host plants to restrict the infection- or nodulation-susceptible zone in a narrow area of the roots; however, mutants defective in this control such as harl and klavier (klv) of L. japonicus cannot arrest further nodulation on young, developing roots. These mutations result in overproduction of nodules, which cover almost entire root regions (i.e., hypernodulation) (Oka-Kira et al. 2005; Wopereis et al. 2000). More impor-
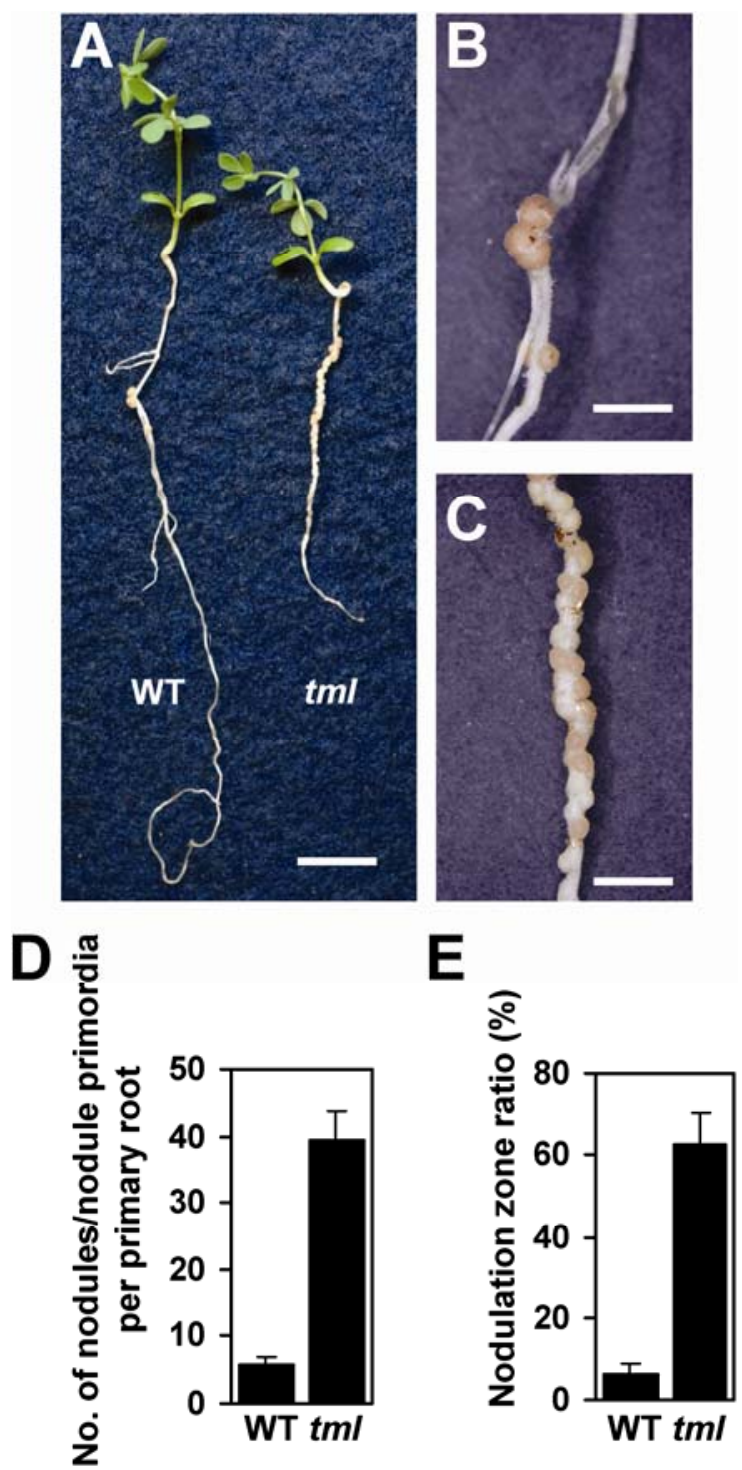

Fig. 1. Hypernodulation phenotype of too much love $(\mathrm{tml})$. Wild-type MG20 (WT) and tml mutants were inoculated with Mesorhizobium lot MAFF303099 4 days after sowing and analyzed at 21 days postinoculation. A, Nodulation phenotype in WT and tml. Scale bar $=1 \mathrm{~cm}$. Close-up view of the nodules developed in $\mathbf{B}$, WT and $\mathbf{C}, t m l$. Scale bar $=2 \mathrm{~mm}$. D, Nodule and nodule primordium number per primary root of WT and tml. $\mathbf{E}$, Nodulation zone ratio of WT and $\mathrm{tml}$. Nodulation zone ratio is the ratio of the nodulated zone length (i.e., the length from the uppermost nodule to the lowermost nodule) to the primary root length. Error bars indicate standard deviation ( $n=15$ to 16 ). tantly, reciprocal grafting experiments using harl or klv mutants and wild-type plants have shown that the hypernodulation phenotype of harl and $k l v$ is determined by the shoot genotype (Krusell et al. 2002; Nishimura et al. 2002; Oka-Kira et al. 2005). This indicates that HARI and $K L V$ have a function in the shoots and that the negative regulation of nodulation requires a long-distance communication between the shoots and the roots. The recent molecular cloning of HAR1 revealed that HARl encodes a CLAVATA1 (CLV1)-like receptor kinase and this gene and its functions are conserved among other legume species, such as M. truncatula, $P$. sativum, and Glycine max (Krusell et al. 2002; Nishimura et al. 2002; Schnabel et al. 2005; Searle et al. 2003). Further expression analysis using a $\beta$-glucuronidase (GUS) reporter gene driven by the HARl native promoter have elucidated that HARl is expressed mainly in the phloem tissues of leaves, stems, roots, and nodules (Nontachaiyapoom et al. 2007). This phloem-specific expression of HARl seems to be reasonable, given that this gene product functions in the shoots for the root nodule number control and that phloem tissues are generally viewed as a conduit for such a long-distance communication (Lough and Lucas 2006; Ruiz-Medrano et al. 2001).

The identification of HARl in L. japonicus and HARI orthologs in other legumes has shed light on the molecular mechanism of the negative feedback regulation of nodulation. However, the exact process still remains unclear and many questions need to be addressed. For example, what kind of signaling molecules and information does HAR1 perceive? What is the molecular property of the potential mobile signals that are generated in the shoots? How is such a mobile signal decoded in the roots? To gain insights into these aspects, isolation and characterization of other hypernodulating mutants are of great significance.

Here, we report a novel hypernodulating mutant named too much love $(\mathrm{tml})$, which was produced by ion beams. We show that the hypernodulation phenotype of $t \mathrm{ml}$ mutants is controlled by the root genotype and that the $t m l$ mutation does not enhance the hypernodulation phenotype of harl mutants. Thus, we propose that HARl and TML constitute the same long-distance signaling pathway that regulates nodule development.

\section{RESULTS}

\section{Isolation of a novel hypernodulating mutant $\mathrm{tml}$.}

In model legume $L$. japonicus, only two hypernodulating mutants, harl and $k l v$, have been characterized (Oka-Kira et al. 2005; Wopereis et al. 2000). To better understand the genetic control of nodulation, we irradiated wild-type MG-20 seed of L. japonicus with carbon-ion beams and screened the $\mathrm{M}_{2}$ progeny for symbiotic mutants which affect nodule development. From this screening, one hypernodulating mutant line was isolated and named $t m l$. The $\mathrm{F}_{1}$ individuals from backcrossing $t m l$ to the parental MG-20 all showed wild-type nodulation and the resulting $\mathrm{F}_{2}$ plants segregated at 58:20 for wildtype and hypernodulation phenotype, a ratio indistinguishable to $3: 1\left(\chi^{2}=0.017, P>0.05\right)$. Therefore, the $t m l$ mutation is recessive monogenic. Moreover, to test whether a $\mathrm{tml}$ mutant is an additional allele of the previously reported hypernodulating mutants, we crossed tml with $k l v$ or harl-7, which is a harl allele that was recently isolated in the MG-20 background (discussed below). However, none of the $\mathrm{F}_{1}$ progeny exhibited hypernodulation, indicating that $t m l$ is very likely to be a novel mutant (data not shown).

\section{Hypernodulation phenotype of $\mathrm{tml}$ mutants.}

The $t m l$ mutants and wild-type plants were inoculated with Mesorhizobium loti MAFF303099 and nodulation phenotypes 
were examined at 21 days post inoculation (dpi). The nodules of $\mathrm{tml}$ were relatively small compared with those of the wild type and were densely developed on the wider range of the roots, while those of wild type were restricted within a narrow region of the roots (Fig. $1 \mathrm{~A}$ through $\mathrm{C}$ ). The $\mathrm{tml}$ mutants produced approximately eight times the number of nodules and nodule primordia formed in wild-type plants (Fig. 1D). We also observed a drastically enhanced nodulation zone ratio of $\mathrm{tml}$, which is the calculated length of the nodulated region normalized to the primary root length (Fig. 1E). These characteristics of $\mathrm{tml}$ hypernodulation are reminiscent of harl and $\mathrm{klv}$ mutants. This suggests that $t m l$ mutants might impair the negative feedback regulation of nodulation. However, unlike harl and $\mathrm{klv}, \mathrm{tml}$ mutants show relatively mild nonsymbiotic phenotypes. In the absence of $M$. loti, tml developed only slightly shorter primary roots with fewer lateral roots than the wild type but otherwise possessed normal plant architecture (data not shown). In contrast, harl mutations result in stunted shoots as well as short roots with increased lateral roots, whereas $k l v$ exhibits pleiotropic phenotypes such as stem fasciation, aberrant leaf veins, late flowering, and so on (Oka-Kira et al. 2005; Wopereis et al. 2000).

To analyze the effects of the $t m l$ mutation more accurately, we inoculated $t m l$ and wild-type plants with M. loti NZP2235 which constitutively expresses the lac $Z$ reporter gene. Roots were excised and stained for $\beta$-galactosidase activity at several time points and infection thread formation, which is a hallmark of early infection, was examined under light microscopy. Wild-type plants reached a plateau in infection thread number in as early as $7 \mathrm{dpi}$, whereas $\mathrm{tml}$ mutants continued to produce more infection threads even at 14 dpi (Fig. 2A). Correlated with this observation, the number of nodules and nodule primordia of the wild type reached a steady level at 7 dpi but it could not stop increasing in $\mathrm{tml}$ (Fig. 2B). Moreover, the infection threads of $t m l$ were distributed in a wider range of roots than those of the wild type (data not shown). This is consistent with the expanded nodulation zone of $t m l$ (Fig. $1 \mathrm{C}$ and E). The wide infection-susceptible zone and the continuous infection in $\mathrm{tml}$ suggest that $\mathrm{tml}$ mutants have a defect in the negative feedback control of nodulation rather than the ethylene-mediated infection arrest, alteration of which does not affect the infection or nodulation-susceptible zone length (Nukui et al. 2004; Penmetsa and Cook 1997).

\section{Ethylene sensitivity of $\mathrm{tml}$ mutants.}

Although the nodulation phenotype of $\mathrm{tml}$ is distinct from that of ethylene-insensitive mutants and transgenic plants (Nukui et al. 2004; Penmetsa and Cook 1997), we cannot rule out the possibility that the ethylene inhibition of nodulation might be partially altered in $t m l$, enhancing its hyperinfection. To test this theory, we first examined ethylene sensitivity of tml mutants using 1-aminocyclopropane-1-carboxylate (ACC), the immediate ethylene precursor. Both wild-type and $t m l$ seedlings grown in the presence of ACC and also in dark conditions showed the typical triple response of ethylene: inhibition of hypocotyl and root elongation, radial hypocotyl swelling, and exaggerated curvature of apical hooks (Fig. 3A) (Guo and Ecker 2004). Further, the hypocotyl growth of $\mathrm{tml}$ was compromised in an ACC-dose-dependent manner indistinguishable from the wild type, indicating that ethylene signaling per se is not impaired in $t m l$ mutants (Fig. 3B).

In addition to the ethylene sensitivity of $t m l$, it seems that the $t m l$ mutation does not affect nodule positioning, which is also regulated by ethylene. Microscopic analysis with transverse root sections revealed that, in wild-type MG-20 plants, the majority of the nodules $(>75 \%)$ were formed opposite the xylem poles (Table 1). This would suggest that the same nodule position control by ethylene as observed in $P$. sativum and $M$. truncatula is conserved in L. japonicus (Heidstra et al. 1997; Penmetsa et al. 2003). This radial nodule positioning pattern was not obviously altered in $\mathrm{tml}$ mutants (Table 1 ). Therefore, together with the proper responses to exogenous ACC, we conclude that $t m l$ mutants do not have a defect in the ethylenemediated negative regulation of nodulation.

\section{Nitrate response of $\mathrm{tml}$ mutants.}

To further investigate the roles of $T M L$, we focused on the effect nitrate has on nodulation in tml. Nitrate is a well-known environmental factor that inhibits nodule formation. It has been shown that mutations in HARl of L. japonicus and HARl orthologs of other legumes result in "nitrate tolerance," exhibiting hypernodulation even in the presence of nitrate (Carroll et al. 1985a and b; Sagan and Duc 1996; Sagan et al. 1995; Schnabel et al. 2005; Wopereis et al. 2000). More recently, it has been elucidated that another hypernodulating mutant, $k l v$, also shows partial nitrate tolerance (Oka-Kira et al. 2005), suggesting that there might be some link between nitrate signaling and the negative feedback control of nodulation.

The wild type, $t m l$, and harl-7 were inoculated with $M$. loti MAFF303099 and grown with or without $\mathrm{KNO}_{3}$ as a nitrate source. In the wild type, $5 \mathrm{mM} \mathrm{KNO}_{3}$ was sufficient to reduce
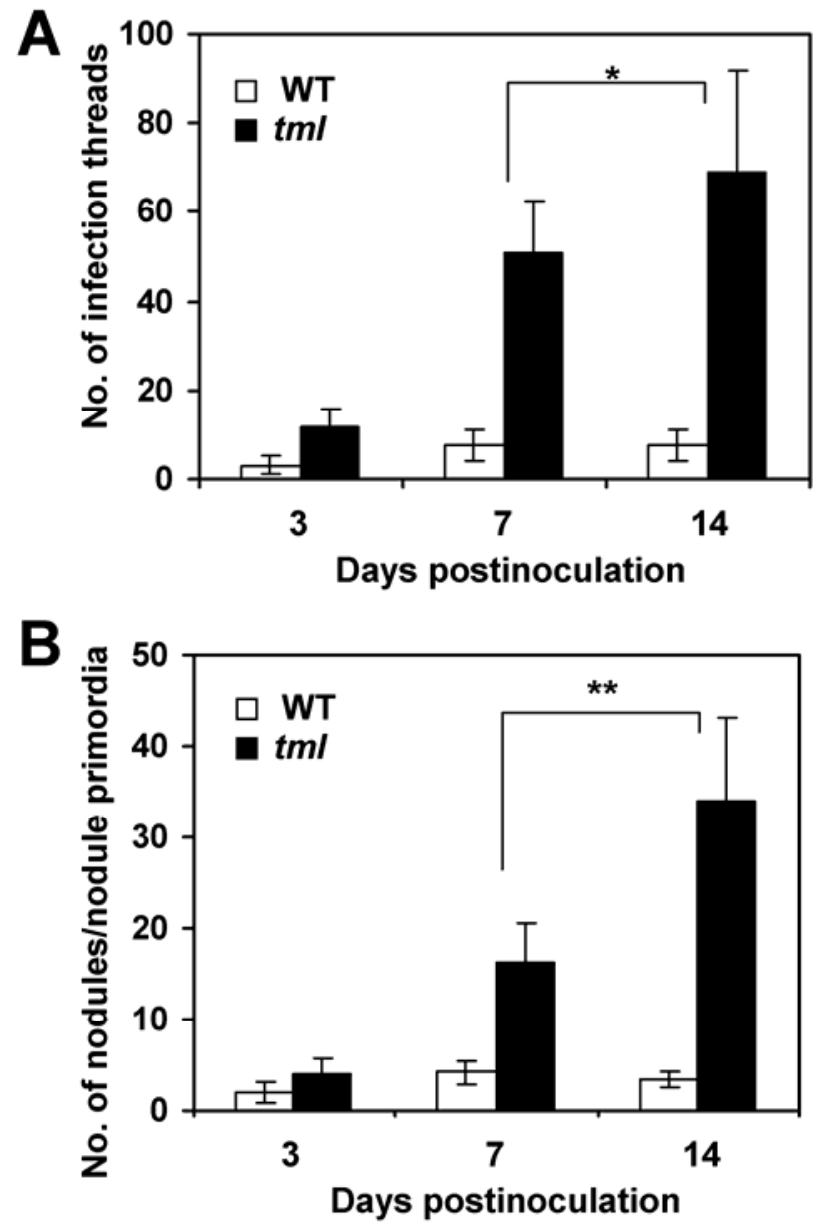

Fig. 2. Early infection events of wild type and too much love ( $\mathrm{tml}$ ). Wild-type MG-20 (WT) and $t m l$ mutants were inoculated 4 days after sowing with Mesorhizobium loti NZP2235 strain, which constitutively expresses the lacZ reporter gene. Roots were stained for $\beta$-galactosidase activity at the indicated time points and $\mathbf{A}$, infection threads and $\mathbf{B}$, nodules and nodule primordia on the primary roots were counted. Error bars indicate standard deviation ( $n=$ 12). Asterisks indicate significant differences between 7 and 14 days postinoculation ( $t$ test; $*$ and $* *$ indicate $P<0.05$ and 0.01 , respectively). 
the nodule and nodule primordium number to approximately $30 \%$ of the nonnitrate control, while neither of $\mathrm{tml}$ or harl-7 showed such a reduction at this $\mathrm{KNO}_{3}$ concentration (Fig. 4B). However, unlike harl-7, the nodule and nodule primordium number of $\mathrm{tml}$ decreased to approximately $50 \%$ when the plants were grown in the presence of $10 \mathrm{mM} \mathrm{KNO}_{3}$ (Fig. 4B), indicating that $\mathrm{tml}$ mutants are only partially insensitive to ni-
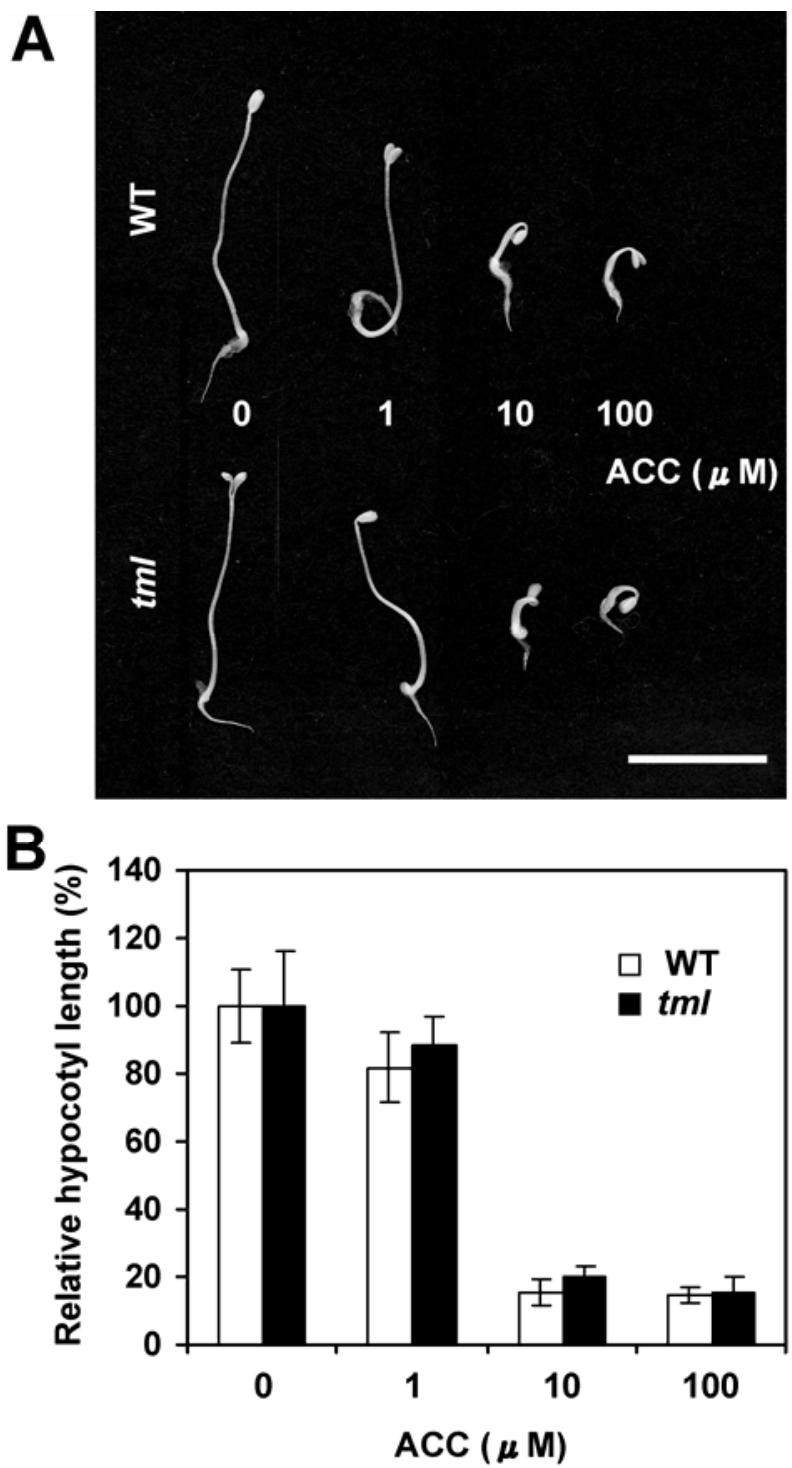

Fig. 3. Ethylene sensitivity of wild type and too much love $(\mathrm{tml})$. Wildtype MG-20 (WT) and $t m l$ seeds were sown on agar plates containing the ethylene precursor 1-aminocyclopropane-1-carboxylate (ACC) (0 to 100 $\mu \mathrm{M})$ and grown vertically in darkness for 7 days. A, Ethylene response observed in WT and $\mathrm{tml}$. Scale bar $=2 \mathrm{~cm}$. B, Relative hypocotyl length of the ACC-treated seedlings. The value of the untreated control was set at $100 \%$. Error bars indicate standard deviation $(n=9$ to 12$)$.

Table 1. Nodule positioning in wild type (WT) and too much love (tml) mutant $^{\mathrm{a}}$

\begin{tabular}{lccc}
\hline & \multicolumn{3}{c}{ No. of nodules (\%) } \\
\cline { 2 - 4 } Genotype & Total $^{\mathbf{b}}$ & Opposite xylem & In between \\
\hline WT (MG-20) & 46 & $35(76.1)$ & $11(23.9)$ \\
$t m l$ & 60 & $44(73.3)$ & $16(26.7)$ \\
\hline
\end{tabular}

a Primary roots of Mesorhizobium loti MAFF303099-inoculated plants were transversely sectioned at 21 days postinoculation and the nodule position relative to the xylem poles was determined under light microscopy.

${ }^{\mathrm{b}}$ Nodules were randomly selected from 10 plants. trate. More importantly, in spite of this attenuated nitrate response in nodulation, the morphology of the nodules and nodule primordia of $t m l$ plants grown with nitrate was compatible with those of the wild type but not harl-7; relatively small and white bumps rather than mature pink nodules were developed in $t m l$ as well as the wild type in the presence of 5 and $10 \mathrm{mM}$ $\mathrm{KNO}_{3}$ (Fig. 4A). This observation suggests that nitrate is still effective in $\mathrm{tml}$ mutants in regard to the inhibition of nodule maturation.

\section{Root genotype determines $\mathbf{t m l}$ hypernodulation.}

Previous studies have shown that the shoot genotype is responsible for both harl and $k l v$ hypernodulation, indicating that $H A R l$ and $K L V$ function in the shoots and both participate in the long-distance control of nodulation (Krusell et al. 2002; Nishimura et al. 2002; Oka-Kira et al. 2005). To locate the potential site of action of $T M L$, we conducted reciprocal grafting experiments with wild-type plants and $t m l$ mutants. Five dayold seedlings were used for shoot-root grafting surgery and the successful grafts were transferred to vermiculite and inoculated with $M$. loti MAFF303099. Grafting a tml shoot onto a wild-type root led to wild-type nodulation (Fig. 5); in contrast, grafting a wild-type shoot onto a $\mathrm{tml}$ root resulted in an increased number of nodules and nodule primordia, which was indistinguishable from that of $t \mathrm{ml}$ self-grafts. This root-determined hypernodulation of $t m l$ indicates that, unlike $H A R l$ and $K L V, T M L$ functions in the roots rather than in the shoots.

The role of $T M L$ in the roots, but not in the shoots, prompted us to ask whether a root factor TML and a shoot factor HARI genetically interact with each other despite the different sites of action. For this purpose, we carried out reciprocal grafting using tml and harl-7 mutants. We confirmed that the hypernodulation of harl-7 is regulated by the shoots, consistent with previous studies using different harl alleles (Fig. 5) (Krusell et al. 2002; Nishimura et al. 2002). This shoot-regulated harl-7 hypernodulation was not obviously enhanced by grafting a harl-7 shoot onto a $t m l$ root (Fig. 5), suggesting that TML and HARl might constitute the same long-distance signaling. On the other hand, grafting a $t m l$ shoot onto a harl-7 root complemented the hypernodulation of each other (Fig. 5), further supporting the specific roles of TML and HARI in the roots and the shoots, respectively.

Based on these findings, at least two potential mechanisms by which a root factor $T M L$ exerts its inhibitory effect on nodulation can be speculated: TML might perceive or mediate an unknown shoot-derived signal produced by HAR1, or TML might generate or relay any root-derived signal that could potentially activate HAR 1 in the shoots. If the former model is the case, the effect of TML should be local and should not be propagated beyond the initial site of action. On the other hand, if the latter model is the case, the effect of $T M L$ could travel from one root to another via a shoot-factor HARl.

To examine which hypothesis is more valid, we designed inverted-Y grafting, where a sliced root is grafted into a short slit made in a stock plant (Fig. 6A). This approach allows us to analyze nodule development on two genetically different roots in the same plant. Using 5-day-old seedlings, we generated wild-type/tml heterografts as well as wild-type and $t m l$ selfgrafts. The assembled grafts were grown in vermiculite for 4 days and then inoculated with $M$. loti MAFF303099. In wildtype/tml heterografts, the wild-type stock roots induced only a small number of nodules ( $1.0 \pm 0.8$ nodules per root) (Fig. 6B, $\mathrm{C}$, and $\mathrm{E}$ ), indicating that the HAR1/TML-mediated negative regulation of nodulation is still functional at least in the stock plants. On the other hand, the $t m l$ inserts developed an excessive number of nodules in the wide root region, which was not restored by the functional $T M L$ in the stock root (Fig. 6B, C, 
A
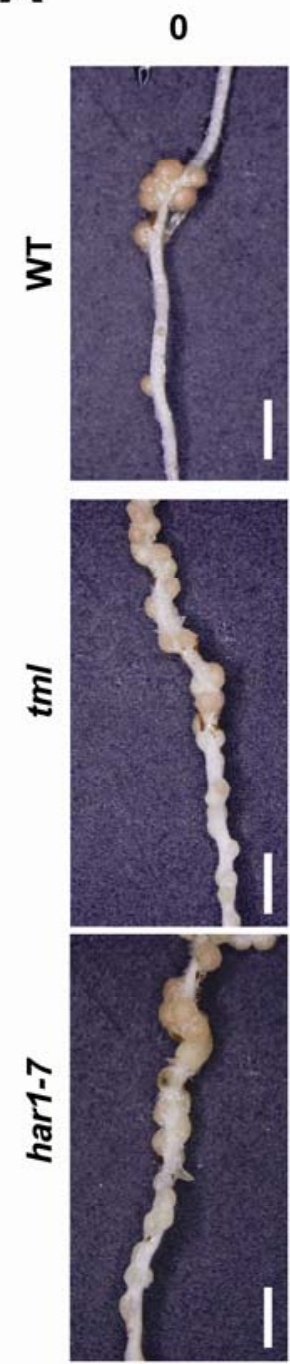

B

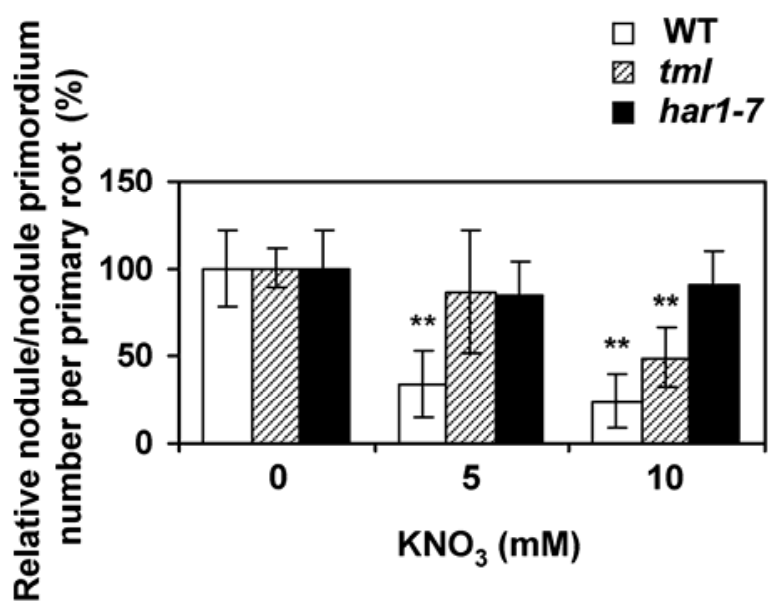

Fig. 4. Nitrate response of wild type, too much love ( $\mathrm{tml}$ ) and har1-7. Wild-type MG-20 (WT), tml, and harl-7 were inoculated with Mesorhizobium loti MAFF303099 4 days after sowing and grown for 21 days in the presence of $\mathrm{KNO}_{3}(0$ to $10 \mathrm{mM})$. A, Nodule development under 0 , 5 , and $10 \mathrm{mM} \mathrm{KNO}$ (from left to right). Scale bars $=2 \mathrm{~mm}$. B, Relative nodule and nodule primordium number per primary root of the $\mathrm{KNO}_{3}$ treated plants. The value of the untreated control was set at $100 \%$. Error bars indicate standard deviation $(n=14$ to 16$)$. Asterisks show significant differences compared with the untreated control $(t$ test; $* *$ indicates $P<0.01)$. and F). Therefore, the effect of $T M L$ is likely to be local, supporting the former hypothesis that TML might function downstream of HAR1, presumably as a receptor or mediator of unknown shoot-derived signal molecules.

\section{Mapping of the $t m l$ mutation.}

To better understand its precise role, we attempted to clone $T M L$. By using simple sequence repeat (SSR) and derived cleaved amplified polymorphic sequence (dCAPS) molecular markers, the $\mathrm{tml}$ mutation was mapped between two markers, SMP002 and TM0356, on the long arm of chromosome 1 (Fig. 7A). We could not further narrow the candidate region in this interval, because there was no additional marker available except TM0064. Nevertheless, based on the recombination frequency, the TML locus was estimated to be only 0.05 centimorgans apart from SMP002. Thus, we assembled a contig from LjT06B17 toward $T M L$ and finally found that $\mathrm{tml}$ has a large deletion ( $>220 \mathrm{~kb}$ ) encompassing at least two TAC/BAC clones, LjT35O15 and LjB302D15 (Fig. 7B). The exact total deletion size is unknown because further chromosome walking was hampered by highly repetitive sequences in the south end of LjB302D15 (data not shown). Polymerase chain reaction (PCR) analysis revealed that all tested hypernodulating lines in the $\mathrm{F}_{2}$ progeny from backcrossing tml to the wild type contained the deletion, indicating that the deletion is tightly linked to $\mathrm{tml}$ hypernodulation phenotype (Fig. 7C). Therefore, we conclude that this large deletion is, indeed, the $t m l$ mutation.

Genetic interaction between $T M L$ and $H A R I$.

By taking advantage of the presence of a deletion in $t \mathrm{ml}$, we generated $\mathrm{tml}$; harl-7 double mutants to examine the possible genetic interaction between TML and HARl. First, we crossed $\mathrm{tml}$ with harl-7 and genotyped the $\mathrm{F}_{2}$ individuals for the $\mathrm{tml}$ deletion as well as the harl-7 mutation. The observed segregation ratio was 32:9:7:4 for the wild type, tml, harl-7, and $\mathrm{tml}$; harl-7 double, fitting the expected ratio of $9: 3: 3: 1\left(\chi^{2}=1.265\right.$, $P>0.05)$. The resulting double-mutant progeny, together with wild-type and single mutants, were then inoculated with $M$.

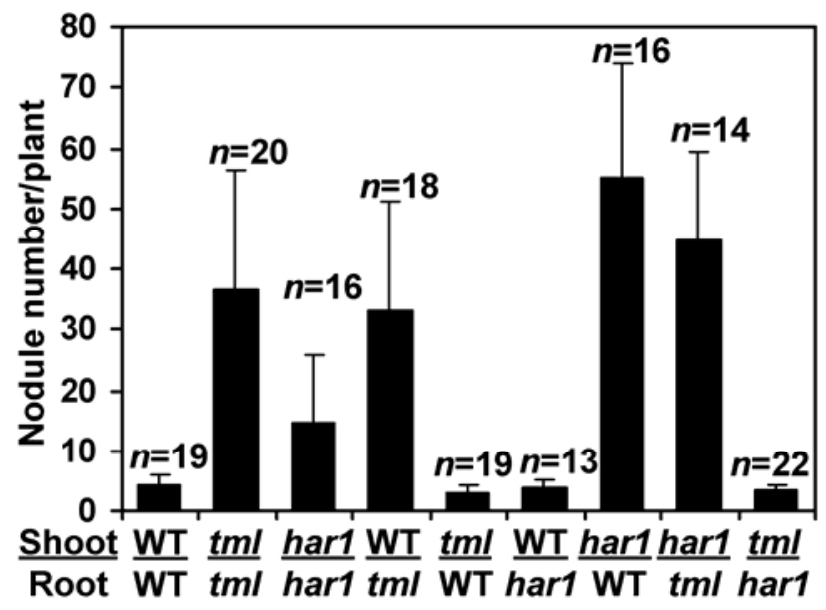

\section{Grafting combination}

Fig. 5. Shoot-root grafting with wild type, too much love (tml) and harl-7. Five day-old seedlings of wild-type MG-20 (WT), tml, and harl-7 were used for shoot-root grafting surgery and grown on moistened filter papers for 7 days. Successful grafts were transferred to vermiculite and inoculated with Mesorhizobium loti MAFF303099. Nodules and nodule primordia per plant were counted at 21 days postinoculation. The shoot genotype and the root genotype used for each grafting combination are shown. Error bars indicate standard deviation. The number of analyzed grafts is provided above each bar. 
loti MAFF303099 and analyzed at $21 \mathrm{dpi}$. The tml:harl-7 double mutants did not show an enhanced nodule and nodule primordium number or nodulation zone ratio, compared with the parental single mutants; hence, the double mutations do not posses any obvious additive effects on nodulation (analysis of variance, $P<0.01$ ) (Table 2). This result indicates that $T M L$ and HARl function in the same genetic pathway, consistent with the conclusion derived from the reciprocal grafting using tml and harl-7 mutants (Fig. 5).

\section{DISCUSSION}

Potential mode of action of TML.

The $t m l$ mutants develop an increased number of nodules over a wide range of root systems (Fig. 1). This typical hypernodulation phenotype suggests that, similar to harl and $k l v$, $\mathrm{tml}$ mutants have a defect in the autoregulatory inhibition of nodule development. Interestingly, reciprocal grafting showed that the root genotype is responsible for $\mathrm{tml}$ hypernodulation (Fig. 5). In P. sativum, it has been reported that nod3 mutants cause such a root-regulated hypernodulation (Postma et al. 1988). However, in the absence of rhizobial inoculation, nod 3 mutants develop compact roots with increased secondary lateral roots, which were not observed in $t m l$ mutants (Postma et al. 1988). Thus, $L j T M L$ and PsNOD3 may not be fully compatible with each other. To analyze this possible relationship, molecular cloning of the two genes and further comparative studies will be necessary.

The reciprocal grafting (Fig. 5) as well as the double-mutant analysis (Table 2) showed that this root-determined hypernodulation of $\mathrm{tml}$ does not have an additive effect on the shootdetermined hypernodulation of harl-7, indicating that $T M L$ and HARl function in the same genetic pathway; hence, $T M L$ as a root factor and HARI as a shoot factor constitute the same long-distance signaling that control nodule development. Further, the inverted-Y grafting results suggest that $T M L$ is likely to act locally in the roots (Fig. 6).

Based on these observations, we propose a possible signaling network of nodule number control, as shown in Figure 8. First, early infection events in one part of the roots rapidly activate a shoot factor HAR1 (a CLV1-like receptor kinase) by generating or transporting a root-derived mobile signal or simply as a result of sink strength change in the roots. Then, activated HAR1 triggers a downstream signaling to induce a shoot-derived mobile signal, which travels down through the phloem to the whole root system. Finally, the mobile signal is perceived or mediated by TML in the roots, repressing further nodulation in younger developing root regions.

It would be reasonable to postulate that TML plays its inhibitory role against any of the NF signaling components that promote nodulation. Among the known positive regulators, the $\mathrm{Ca}^{2+} /$ calmodulin-dependent protein kinase (CCaMK) and a cy-

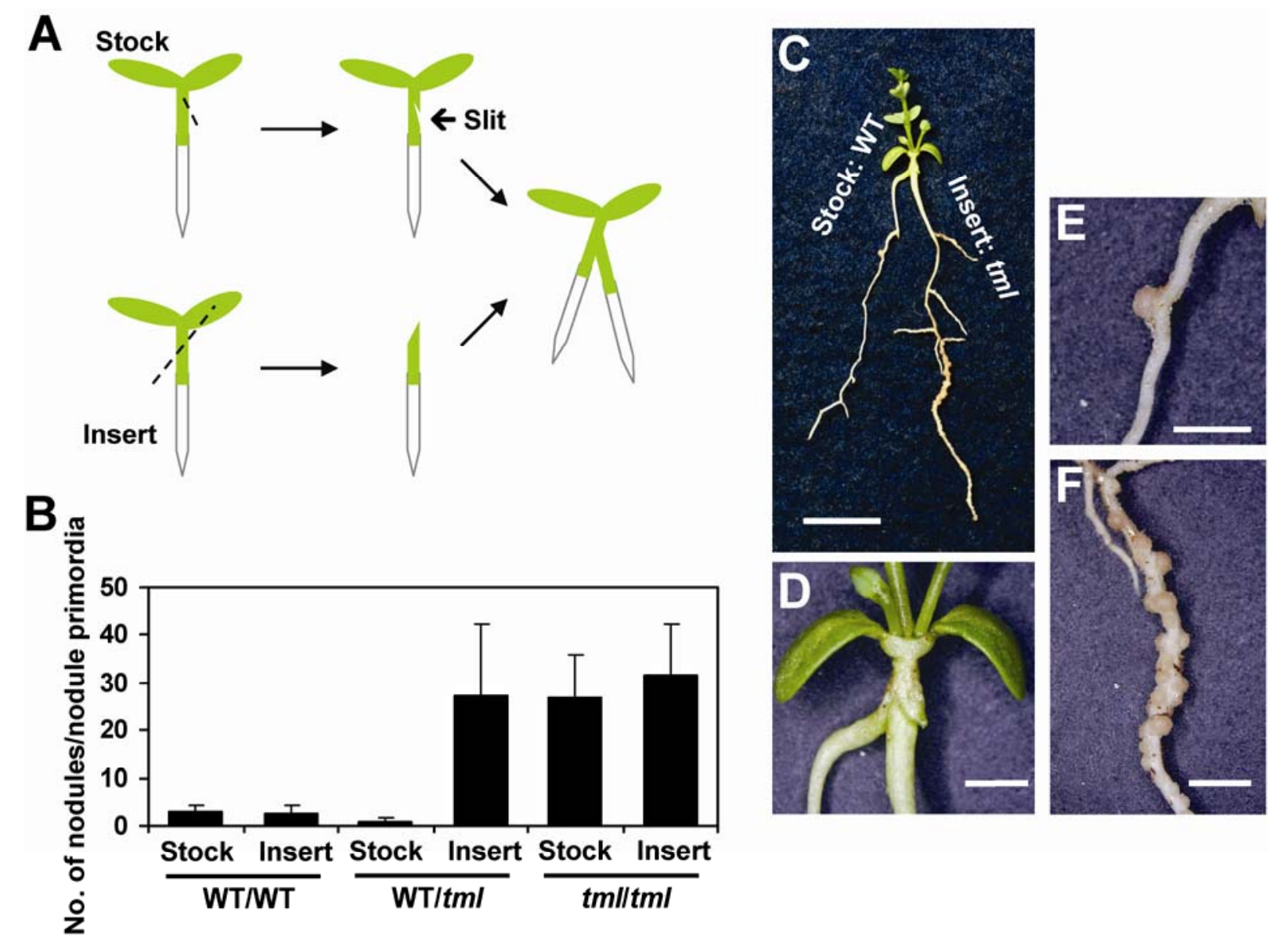

Fig. 6. Inverted-Y grafting with wild type and too much love ( $\mathrm{tml}$ ). A, Schematic overview of inverted-Y grafting. Five-day-old seedlings of wild-type MG20 (WT) and $t m l$ were used for inverted-Y grafting surgery, in which a sliced root was inserted into a short slit made in the side of a stock hypocotyl. Processed grafts were grown on moistened filter papers for 5 days. Successful grafts were then transferred to vermiculite and inoculated with Mesorhizobium loti MAFF303099 after 4 days. B, Number of nodules or nodule primordia developed on the stock roots and the insert roots at 21 days postinoculation (dpi). Each grafting combination is shown as stock/insert. Error bars indicate standard deviation $(n=6$ to 8$)$. $\mathbf{C}$ through $\mathbf{F}$, Nodulation in a WT/tml heterograft at 21 dpi. D, Close-up view of the graft union. E, Close-up view of a nodule formed on the WT stock root. F, Close-up view of nodules and nodule primordia formed on the $\mathrm{tml}$ insert root. Scale bar $=1 \mathrm{~cm}(\mathrm{C})$ or $2 \mathrm{~mm}$ (D through F). 
tokinin receptor LHK1 as well as their upstream components are unlikely to be the TML targets. It has been shown that a gain-of-function mutation in either of the two genes leads to nodule development even in the absence of rhizobia or the NF receptor (i.e., spontaneous nodulation), and that the spontaneous nodule number can be further increased by a harl mutation (Tirichine et al. 2006a and b; Tirichine et al. 2007). These results suggest that the HARl-mediated autoregulatory inhibition of nodulation is still intact in these two mutants. Therefore, the HAR1-TML scheme might repress any other possible positive regulator that acts downstream of CCaMK and LHK1 in the NF-signaling cascade.

Although the HAR1-to-TML long-distance signaling model proposed here is simple and attractive, some cautions should be observed. For example, the local effect of TML on nodulation was inferred only from the inverted-Y grafting results. It is still possible that the effects of TML could be systemic (i.e., $T M L$ acts upstream of $H A R l$ ) but such a systemic repression of nodulation could not be detected due to incomplete or delayed grafting connections. More importantly, considering that a large genomic region (>220 kb) is deleted in $t m l$ (Fig. 7B), we cannot exclude the possibility that the $t m l$ hypernodulation

Table 2. Genetic interaction between TOO MUCH LOVE (TML) and HARI

\begin{tabular}{llcc}
\hline Genotype & $\boldsymbol{n}$ & $\begin{array}{c}\text { Nodule/nodule } \\
\text { primordium number }\end{array}$ & $\begin{array}{c}\text { Nodulation zone } \\
\text { ratio (\%) }\end{array}$ \\
\hline WT & 24 & $4.3 \pm 1.3$ & $5.4 \pm 3.2$ \\
tml & 24 & $32.3 \pm 7.4$ & $62.0 \pm 11.4$ \\
harl-7 & 24 & $21.0 \pm 6.5$ & $72.1 \pm 11.0$ \\
tml; harl-7 & 24 & $26.0 \pm 5.6$ & $74.3 \pm 13.2$ \\
\hline
\end{tabular}

a Plants were inoculated with Mesorhizobium loti MAFF303099 4 days after sowing and nodules and nodule primordia on the primary roots were counted at 21 days postinoculation. Nodulation zone ratio refers to the ratio of the nodulated zone length to the primary root length. WT = wild type. phenotype might be a consequence of a multiple gene loss rather than an alteration in only a single locus. If this is the case, the actual long-distance signaling network would be more complex and would not be easily untangled.

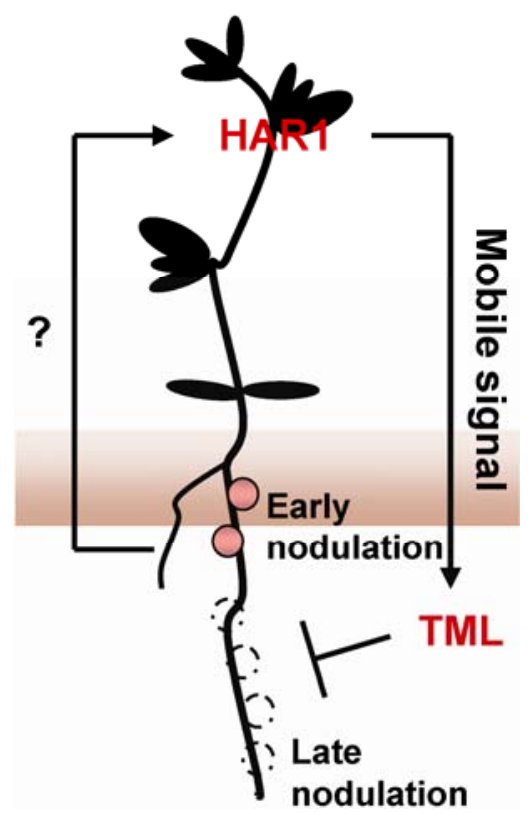

Fig. 8. Model for the long-distance signaling in nodulation. Early nodulation events result in production of an unknown root-derived signal molecule or an alteration of sink strength, which leads to activation of HAR1 in the shoots. The activated HAR1 then generates a mobile signal molecule, which is loaded into the phloem and transported to the entire root system. The shoot-derived signal molecule is perceived or relayed by TOO MUCH LOVE (TML) in the roots, which leads to repression of late nodulation in younger root region.

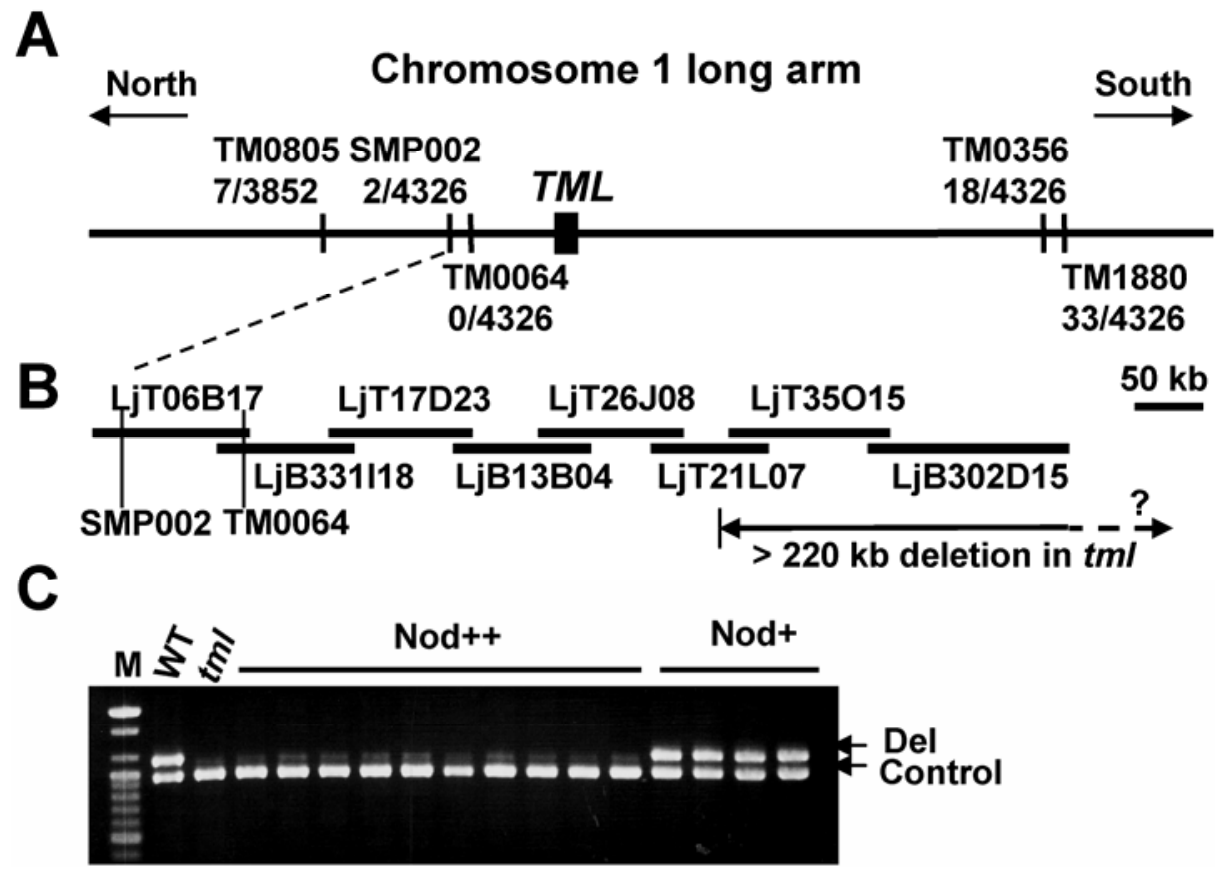

Fig. 7. Mapping of the too much love ( $\mathrm{tml}$ ) mutation. A, Genetic map of the $t \mathrm{ml}$ mutation on the long arm of chromosome 1 . The names of the molecular markers are shown. Values indicate the number of recombination events between each marker and the tml/TML phenotype per number of chromosomes analyzed. B, Physical map of the TAC/BAC clones. The names of the clones are shown. Arrow indicates an approximate deletion found in the tml genome. $\mathbf{C}$, Linkage between the $\mathrm{tml}$ deletion and the hypernodulation phenotype. The $\mathrm{tml}$ mutant was backcrossed to the wild type (WT) and the $\mathrm{F}_{2}$ progeny was subjected to a multiplex polymerase chain reaction analysis. Primers designed on the north end of LjT35O15 were used to detect the tml deletion (Del) and HARl-specific primers were used as a control. All tested hypernodulating lines (Nod++) contained the deletion, whereas WT lines (Nod+) did not. M, DNA size marker. 
Link between the nitrate inhibition and the HAR1/TML signaling.

It has long been known that exogenous nitrate has an inhibitory effect on nodule development, its growth, and its activity (Carroll and Mathews 1990) but is ineffective in harl hypernodulating mutants of $L$. japonicus and other legume mutants that have mutations in HARl orthologs (Carroll et al. 1985a and b; Sagan et al. 1995; Sagan and Duc 1996; Schnabel et al. 2005; Wopereis et al. 2000). In addition to harl, tml hypernodulating mutants also show partial insensitivity to nitrate (Fig. 4). Based on this concurrence of nitrate tolerance and hypernodulation, it can be speculated that nitrate might repress nodulation by activating the $H A R l$-involved long-distance signaling pathway; hence, the nitrate effects should be systemic. Consistent with this prediction, it has been shown that the nitrate tolerance of $n t s 382$ and en6500 hypernodulating mutants, which have mutations in the HARl ortholog of G. max, is determined by the shoot genotype (Day et al. 1989; Francisco and Akao 1993). However, contrary to this observation, the previous split-root experiments with soybean have revealed that nitrate application to a half portion of roots can inhibit nodulation only in the treated roots but not in the nontreated root half, supporting the local effects of nitrate (Cho and Harper 1991). Therefore, it seems that the nitrate response on nodulation cannot be explained simply by activation of the HARI/TML-mediated autoregulatory signaling. Rather, nitrate signaling should be more complex and might have multiple sites of action on several nodule developmental stages. Because $\mathrm{tml}$ mutants showed partial nitrate insensitivity only for nodule and nodule primordium number, but not for nodule maturation (Fig. 4), potential nitrate signaling might be branched into at least two pathways, which separately repress nodule initiation and nodule growth. It appears that $t m l$ has a defect only in the former process, whereas harl has an alteration in both of the pathways. Further phenotypic and genetic analyses using $t m l$ and harl should provide us with clues for more precise modes of action regarding nitrate in nodulation.

\section{The $t m l$ mutant as a novel reagent} to dissect the signaling network of nodule number control.

Despite the uncertainty of the molecular property of TML, tml mutants can be a strong genetic tool to identify an unknown shoot-derived signal molecule, which is possibly generated in a $H A R l$-dependent manner. If root factor $T M L$ functions downstream of HARl as we propose, induction of such a mobile signal should be impaired only in harl but not in $\mathrm{tml}$ mutants. Therefore, it would be possible to isolate the potential signal molecule candidates by a comparative transcriptome or proteome analysis focusing on tml and harl mutants. Moreover, identification of mutants that suppress $t \mathrm{ml}$ hypernodulation might allow us to better understand the crosstalk of the NF pathway and the HAR1/TML-mediated autoregulatory signaling. Considering these applications of $\mathrm{tml}$ mutants for future studies, "too much love" might not always be a disaster.

\section{MATERIALS AND METHODS}

\section{Plant materials and growth conditions.}

The tml mutant was produced by carbon-ion beams. Ionbeam irradiation was carried out as previously described (OkaKira et al. 2005; Tanaka et al. 1997). The energy of carbon ions was $320 \mathrm{MeV}$ and the mean LET within the seed was estimated to be $86 \mathrm{keV} / \mu \mathrm{m}$. Approximately 3,300 dry seeds of wild-type MG-20 of L. japonicus were irradiated with carbon ions at a dose of $80 \mathrm{~Gy}$. Approximately 35,000 of the resulting $\mathrm{M}_{2}$ seed were screened for the hypernodulation phenotype.
The harl-7 mutant of MG-20 was isolated by ethylmethane sulfonate mutagenesis. The harl-7 mutation is a nonsense mutation in the leucine-rich repeat domain of HAR1 (TGG to TGA; Trp346 to stop).

For tml;harl-7 double mutants were obtained by crossing tml with harl-7 and genotyping the $\mathrm{F}_{2}$ population by PCR. Primers used for the $t m l$ deletion were 5'-TCAGGCAAT TGTTGGCTATATG-3' and 5'-TCATCACCAAGAGATTTGA ACG-3', which amplify a 1,157-bp region within the deletion. Primers used for the PCR control were 5'-GAATCAGAGT GTCTTACTTGTTAGTGC-3' and 5'-TTTGGAAGAAGTTCA TTAGAGTGAGA-3', which amplify a 972-bp region within HAR1. The dCAPS markers used for harl-7 genotyping are 5'CAAATCTCGAAACGCTTCAGGCTTG-3' and 5' TGAGTGACCTACACTCGCCGATT-3', which amplify a 225bp region spanning the harl-7 mutation. The PCR products were digested with $S m l$ I (New England BioLabs, Ipswich, MA, U.S.A.), which results in cutting (203 and 22 bp) only in harl-7.

In general, plant seed were sown in vermiculite (VS-kakou) in plastic pots after overnight imbibition and grown under a cycle of light and darkness of 16 and $8 \mathrm{~h}$, respectively, at a light intensity of $150 \mu \mathrm{E} / \mathrm{s} / \mathrm{m}^{2}$ at $22^{\circ} \mathrm{C}$ in a Biotron LH-300 (Nihon-ika Co. Ltd., Osaka, Japan) incubator.

For ethylene-sensitivity assay, surface-sterilized seed were sown on $0.8 \%$ agar plates containing B\&D (Broughton and Dilworth 1971) and ACC (0 to $100 \mu \mathrm{M})$ and grown vertically in darkness, but otherwise in the same growth conditions as above. Etiolated seedlings were examined 7 days after sowing.

\section{Nodulation assay.}

M. loti MAFF303099 or NZP2235 variants expressing the lacZ reporter gene under hemA promoter were cultured in yeast extract-manitol liquid media for two overnights. The harvested bacterial cells were resuspended at a dilution of 1:200 with $\mathrm{B} \& \mathrm{D}$ solution and inoculated in plants by moistening vermiculite homogenously 4 days after sowing. Plants were harvested at $21 \mathrm{dpi}$ or as otherwise noted and all nodules and nodule primordia visible to unaided eyes were counted. For a nitrate response assay, 5 or $10 \mathrm{mM} \mathrm{KNO}_{3}$ was supplemented in $\mathrm{B} \& \mathrm{D}$.

For microscopic analysis of early infection events, plants were harvested either 3,7 , or 14 days after M. loti NZP2235 inoculation. Excised roots were fixed with $1.25 \%$ glutaraldehyde in $0.2 \mathrm{M}$ sodium phosphate buffer ( $\mathrm{pH}$ 7.0) for at least $1 \mathrm{~h}$ by vacuum infiltration and stained for $\beta$-galactosidase activity with a staining solution containing $0.08 \%$ 5-bromo-4-chloro-3indolyl- $\beta$-D-galactopyranoside, $5 \mathrm{mM} \mathrm{K}_{3}\left(\mathrm{Fe}(\mathrm{CN})_{6}\right)$, and $5 \mathrm{mM}$ $\mathrm{K}_{4}\left(\mathrm{Fe}(\mathrm{CN})_{6}\right)$. The roots were incubated in the same solution overnight at room temperature. Stained roots were washed with $0.2 \mathrm{M}$ sodium phosphate buffer ( $\mathrm{pH} 7.0)$ several times and examined under bright-field microscopy (Olympus, Tokyo).

\section{Microscopic analysis of nodule positioning.}

Plants were inoculated with M. loti MAFF303099 and harvested at $21 \mathrm{dpi}$. Primary root regions harboring nodules were dissected into approximately $1-\mathrm{cm}$ lengths by hand and embedded in 5\% agar. Transverse sections of the nodules were processed into $100-\mu \mathrm{m}$ thickness by a microslicer (Dosaka). Four to six sections per nodule were obtained and the nodule positions relative to the xylem poles were determined under bright-field light microscopy (Olympus).

\section{Grafting.}

Seeds were sown on moistened no. 2 filter papers (Whatman), which were placed in plastic square plates and grown vertically for 2 days in darkness and 3 days in light/dark 
cycles. For shoot-root grafting, the Arabidopsis wedge-grafting protocol (Turnbull et al. 2002) was modified for L. japonicus. First, 5-day-old seedlings were cut perpendicularly at the hypocotyls with a no. 15 scalpel blade. A shoot scion was then sliced at an angle and inserted into a short vertical slit (approximately $2 \mathrm{~mm}$ ) made on a rootstock with forceps under a stereomicroscope (Olympus). Grafts were grown horizontally on the filter papers in plastic plates for 7 days under regular growth conditions and transferred to vermiculite supplemented with B\&D. The grafts were inoculated with $M$.loti MAFF303099 2 days after transferring and the nodules and nodule primordia per plant were counted at $21 \mathrm{dpi}$. Unsuccessful grafts which showed retarded growth or yellowing were discarded.

For inverted-Y grafting, a root was sliced diagonally at the hypocotyl for an insert, while a short slit (1 to $2 \mathrm{~mm}$ ) was made on the side of the hypocotyl of the stock seedling. The former root was grafted upward into this slit so that the cut surface of the insert faced the pith of the stock (Fig. 6A). Grafts were grown horizontally on filter papers for 5 days and the roots of the stock plants were trimmed approximately in half to stimulate the inserted root growth. Grafts were then transferred to vermiculite supplemented with $B \& D$. After 4 days of transferring, plants were inoculated with $M$. loti MAFF303099, and nodules and nodule primordia on both the insert roots and the stock roots were counted at $21 \mathrm{dpi}$.

\section{Mapping.}

The $t m l$ mutant was crossed with B-129 of L. japonicus. Genomic DNA was extracted from the cotyledons of the $F_{2}$ individuals and analyzed by PCR with SSR and dCAPS markers. The markers used for high-resolution mapping are as follows: TM0316, TM0805, SMP002, TM0064, TM0356, and TM1880. The SMP002 primers were $5^{\prime}$-GCCTTGAAGAAAACAACA ACG-3' and 5'-GCAACCCACCACAGGTTTTA-3'. Information on the other markers is available at Kazusa DNA Research Institute. Nodulation phenotype of the $\mathrm{F}_{2}$ individuals was determined 21 days after M. loti MAFF303099 inoculation and the phenotypes of the recombinant lines were further confirmed in the $F_{3}$ progeny. Sequence data of the BAC/TAC clones found in this article were deposited in GenBank under accession numbers AP004905, AP004906, AP010919, AP010920, AP010921, AP010922, AP010923, and AP010924.

\section{ACKNOWLEDGMENTS}

We thank N. Suganuma (Aichi University of Education) for providing har1-7 seed, S. Tomisawa for initial characterization of har1-7, and M. Notaguchi (Kyoto University) for technical advice on grafting. We also thank M. Nauerth for proofreading the manuscript. This work was supported by Grant-in-Aid for Science Research for Priority Areas (18056004) and Special Coordination Funds for Promoting Science and Technology from the Ministry of Education, Culture, Sports, Science and Technology, Japan. S. Magori was funded in part by Grant-in-Aid for JSPS Fellows.

\section{LITERATURE CITED}

Broughton, W. J., and Dilworth, M. Y. 1971. Control of leghemoglobin synthesis in snake bean. Biochem. J. 125:1075-1080.

Carroll, B. J., and Mathews, A. 1990. Nitrate inhibition of nodulation in legumes. Pages 159-180 in: Molecular Biology of Symbiotic Nitrogen Fixation. P. Gresshoff, ed. CRS Press Inc, Boca Raton, FL, U.S.A.

Carroll, B. J., McNeil, D. L., and Gresshoff, P. M. 1985a. A supernodulation and nitrate-tolerant symbiotic (nts) soybean mutant. Plant Physiol. 78:34-40.

Carroll, B. J., McNeil, D. L., and Gresshoff, P. M. 1985b. Isolation and properties of soybean [Glycine $\max (\mathrm{L}$.) Merr.] mutants that nodulate in the presence of high nitrate concentrations. Proc. Natl. Acad. Sci. U.S.A. 82:4162-4166.
Cho, M. J., and Harper, J. E. 1991. Effect of localized nitrate application on isoflavonoid concentration and nodulation in split-root systems of wild-type and nodulation-mutant soybean plants. Plant Physiol. 95:1106-1112.

Day, D. A., Carroll, B. J., Delves, A. C., and Gresshoff, P. M. 1989. Relationship between autoregulation and nitrate inhibition of nodulation in soybeans. Physiol. Plant. 75:37-42.

Francisco, P. B., and Akao, S. 1993. Autoregulation and nitrate inhibition of nodule formation in soybean cv. Enrei and its nodulation mutants. J. Exp. Bot. 44:547-553.

Geurts, R., Fedorova, E., and Bisseling, T. 2005. Nod factor signaling genes and their function in the early stages of Rhizobium infection. Curr. Opin. Plant Biol. 8:346-352.

Guo, H., and Ecker, J. R. 2004. The ethylene signaling pathway: New insights. Curr. Opin. Plant Biol. 7:40-49.

Heidstra, R., Yang, W. C., Yalcin, Y., Peck, S., Emons, A. M., van Kammen, A., and Bisseling, T. 1997. Ethylene provides positional information on cortical cell division but is not involved in Nod factorinduced root hair tip growth in Rhizobium-legume interaction. Development 124:1781-1787.

Kosslak, R. M., and Bohlool, B. B. 1984. Suppression of nodule development of one side of a split-root system of soybeans caused by prior inoculation of the other side. Plant Physiol. 75:125-130.

Krusell, L., Madsen, L. H., Sato, S., Aubert, G., Genua, A., Szczyglowski, K., Duc, G., Kaneko, T., Tabata, S., de Bruijn, F., Pajuelo, E., Sandal, N., and Stougaard, J. 2002. Shoot control of root development and nodulation is mediated by a receptor-like kinase. Nature 420:422-426.

Lough, T. J., and Lucas, W. J. 2006. Integrative plant biology: Role of phloem long-distance macromolecular trafficking. Annu. Rev. Plant Biol. 57:203-232.

Madsen, E. B., Madsen, L. H., Radutoiu, S., Olbryt, M., Rakwalska, M., Szczyglowski, K., Sato, S., Kaneko, T., Tabata, S., Sandal, N., and Stougaard, J. 2003. A receptor kinase gene of the LysM type is involved in legume perception of rhizobial signals. Nature 425:637-640.

Malik, N. S., and Bauer, W. D. 1988. When does the self-regulatory response elicited in soybean root after inoculation occur? Plant Physiol. 88:537-539.

Nishimura, R., Hayashi, M., Wu, G. J., Kouchi, H., Imaizumi-Anraku, H., Murakami, Y., Kawasaki, S., Akao, S., Ohmori, M., Nagasawa, M., Harada, K., and Kawaguchi M. 2002. HAR1 mediates systemic regulation of symbiotic organ development. Nature 420:426-429.

Nontachaiyapoom, S., Scott, P. T., Men, A. E., Kinkema, M., Schenk, P. M., and Gresshoff, P. M. 2007. Promoters of orthologous Glycine max and Lotus japonicus nodulation autoregulation genes interchangeably drive phloem-specific expression in transgenic plants. Mol. PlantMicrobe Interact. 20:769-780.

Nukui, N., Ezura, H., Yuhashi, K., Yasuta, T., and Minamisawa, K. 2000. Effects of ethylene precursor and inhibitors for ethylene biosynthesis and perception on nodulation in Lotus japonicus and Macroptilium atropurpureum. Plant Cell Physiol. 41:893-897.

Nukui, N., Ezura, H., and Minamisawa, K. 2004. Transgenic Lotus japonicus with an ethylene receptor gene $\mathrm{Cm}$-ERS1/H7OA enhances formation of infection threads and nodule primordia. Plant Cell Physiol. 45:427435.

Nutman, P. S. 1952. Studies on the physiology of nodule formation. III. Experiments on the excision of root-tips and nodules. Ann. Bot. 16:79101.

Oka-Kira, E., Tateno, K., Miura, K., Haga, T., Hayashi, M., Harada, K., Sato, S., Tabata, S., Shikazono, N., Tanaka, A., Watanabe, Y., Fukuhara, I., Nagata, T., and Kawaguchi, M. 2005. klavier (klv), a novel hypernodulation mutant of Lotus japonicus affected in vascular tissue organization and floral induction. Plant J. 44:505-515.

Penmetsa, R. V., and Cook, D. R. 1997. A legume ethylene-insensitive mutant hyperinfected by its rhizobial symbiont. Science 275:527-530.

Penmetsa, R. V., Frugoli, J. A., Smith, L. S., Long, S. R., and Cook, D. R. 2003. Dual genetic pathways controlling nodule number in Medicago truncatula. Plant Physiol. 131:998-1008.

Pierce, M., and Bauer, W. D. 1983. A rapid regulatory response governing nodulation in soybean. Plant Physiol. 73:286-290.

Postma, J. G., Jacobsen, E., and Feenstra, W. 1988. Three pea mutants with an altered nodulation studied by genetic analysis and grafting. J. Plant Physiol. 132:424-430.

Radutoiu, S., Madsen, L. H., Madsen, E. B., Felle, H. H., Umehara, Y., Grønlund, M., Sato, S., Nakamura, Y., Tabata, S., Sandal, N., and Stougaard, J. 2003. Plant recognition of symbiotic bacteria requires two LysM receptor-like kinases. Nature 425:585-592.

Riely, B. K., Ané, J. M., Penmetsa, R. V., and Cook, D. R. 2004. Genetic and genomic analysis in model legumes bring Nod-factor signaling to center stage. Curr. Opin. Plant Biol. 7:408-413.

Ruiz-Medrano, R., Xoconostle-Cázares, B., and Lucas, W. J. 2001. The 
phloem as a conduit for inter-organ communication. Curr. Opin. Plant Biol. 4:202-209.

Sagan, M., and Duc, G. 1996. Sym28 and Sym29, two new genes involved in regulation of nodulation in pea (Pisum sativum L.). Symbiosis 20:229-245.

Sagan, M., Morandi, D., Tarenghi, E., and Duc, G. 1995. Selection of nodulation and mycorrhizal mutants in the model plant Medicago truncatula (Gaertn.) after g-ray mutagenesis. Plant Sci. 111:63-71.

Schnabel, E., Journet, E. P., de Carvalho-Niebel, F., Duc, G., and Frugoli, J. 2005. The Medicago truncatula SUNN gene encodes a CLV1-like leucine-rich repeat receptor kinase that regulates nodule number and root length. Plant Mol. Biol. 58:809-822.

Searle, I. R., Men, A. E., Laniya, T. S., Buzas, D. M., Iturbe-Ormaetxe, I., Carroll, B. J., and Gresshoff, P. M. 2003. Long-distance signaling in nodulation directed by a CLAVATA1-like receptor kinase. Science 299:109-112.

Stacey, G., Libault, M., Brechenmacher, L., Wan, J., and May, G. D. 2006. Genetics and functional genomics of legume nodulation. Curr. Opin. Plant Biol. 9:110-121.

Sugawara, M., Okazaki, S., Nukui, N., Ezura, H., Mitsui, H., and Minamisawa, K. 2006. Rhizobitoxine modulates plant-microbe interactions by ethylene inhibition. Biotechnol. Adv. 24:382-388.

Tanaka, A., Shikazono, N., Yokota, Y., Watanabe, H., and Tano, S. 1997. Effects of heavy ions on the germination and survival of Arabidopsis thaliana. Int. J. Radiat. Biol. 72:121-127.

Tirichine, L., Imaizumi-Anraku, H., Yoshida, S., Murakami, Y., Madsen,
L. H., Miwa, H., Nakagawa, T., Sandal, N., Albrektsen, A. S Kawaguchi, M., Downie, A., Sato, S., Tabata, S., Kouchi, H., Parniske, M., Kawasaki, S., and Stougaard, J. 2006a. Deregulation of a $\mathrm{Ca} 2+/$ calmodulin-dependent kinase leads to spontaneous nodule development. Nature 441:1153-1156.

Tirichine, L., James, E. K., Sandal, N., and Stougaard, J. 2006b. Spontaneous root-nodule formation in the model legume Lotus japonicus: A novel class of mutants nodulates in the absence of rhizobia. Mol. PlantMicrobe. Interact. 19:373-382.

Tirichine, L., Sandal, N., Madsen, L. H., Radutoiu, S., Albrektsen, A. S., Sato, S., Asamizu, E., Tabata, S., and Stougaard J. 2007. A gain-offunction mutation in a cytokinin receptor triggers spontaneous root nodule organogenesis. Science 315:104-107.

Turnbull, C. G., Booker, J. P., and Leyser, H. M. 2002. Micrografting techniques for testing long-distance signalling in Arabidopsis. Plant J. 32:255-262.

Wopereis, J., Pajuelo, E., Dazzo, F. B., Jiang, Q., Gresshoff, P. M., De Bruijn, F. J., Stougaard, J., and Szczyglowski, K. 2000. Short root mutant of Lotus japonicus with a dramatically altered symbiotic phenotype. Plant J. 23:97-114.

\section{AUTHOR-RECOMMENDED INTERNET RESOURCE}

Kazusa DNA Research Institute Lotus japonicus webpage: www.kazusa.or.jp/lotus/index.html 